Portland State University

PDXScholar

$2-1-2008$

\title{
Templated biomimetic multifunctional coatings
}

\author{
Chih-Hung Sun \\ University of Florida \\ Adriel Gonzalez \\ University of Florida \\ Nicholas C. Linn \\ University of Florida \\ Peng Jiang \\ University of Florida \\ Bin Jiang \\ Portland State University
}

Follow this and additional works at: https://pdxscholar.library.pdx.edu/mth_fac

Part of the Mathematics Commons

Let us know how access to this document benefits you.

\section{Citation Details}

Sun, C., Gonzalez, A., Linn, N. C., Jiang, P., \& Jiang, B. (2008). Templated biomimetic multifunctional coatings. Applied Physics Letters, 92(5), 051107.

This Article is brought to you for free and open access. It has been accepted for inclusion in Mathematics and Statistics Faculty Publications and Presentations by an authorized administrator of PDXScholar. Please contact us if we can make this document more accessible: pdxscholar@pdx.edu. 


\title{
Templated biomimetic multifunctional coatings
}

\author{
Chih-Hung Sun, ${ }^{1}$ Adriel Gonzalez, ${ }^{1}$ Nicholas C. Linn, ${ }^{1}$ Peng Jiang, ${ }^{1, a)}$ and Bin Jiang ${ }^{2}$ \\ ${ }^{1}$ Department of Chemical Engineering, University of Florida, Gainesville, Florida 32611, USA \\ ${ }^{2}$ Department of Mathematics and Statistics, Portland State University, Portland, Oregon 97201, USA
}

(Received 19 September 2007; accepted 20 January 2008; published online 6 February 2008)

\begin{abstract}
We report a bioinspired templating technique for fabricating multifunctional optical coatings that mimic both unique functionalities of antireflective moth eyes and superhydrophobic cicada wings. Subwavelength-structured fluoropolymer nipple arrays are created by a soft-lithography-like process. The utilization of fluoropolymers simultaneously enhances the antireflective performance and the hydrophobicity of the replicated films. The specular reflectivity matches the optical simulation using a thin-film multilayer model. The dependence of the size and the crystalline ordering of the replicated nipples on the resulting antireflective properties have also been investigated by experiment and modeling. These biomimetic materials may find important technological application in self-cleaning antireflection coatings. (C) 2008 American Institute of Physics. [DOI: 10.1063/1.2841818]
\end{abstract}

The natural world is providing enormous inspiration for scientists to mimic the unique functionalities of biological systems for practical applications. ${ }^{1-4}$ For instance, the microstructured cornea of some nocturnal moths can greatly reduce reflection by creating a refractive index gradient across the interface between air and cornea, ${ }^{5,6}$ motivating the production of moth-eye antireflection (or antiglaring) coatings that are widely used in flat-panel displays, car dashboards, optical elements, and solar cell collectors. ${ }^{7,8}$ The selfcleaning effect on the wings of cicada induced by surface microstructures has inspired great interest in creating superhydrophobic coatings to prevent contamination, erosion, and bacterial accumulation. ${ }^{9}$ Although the natural microstructures on moth eyes and cicada wings lead to different biological functionalities, they show remarkable structural similarity, both consisting of non-close-packed (ncp), hexagonally ordered nipple-shaped protrusions with sub300-nm-scale dimensions. ${ }^{6,9}$ This similarity has inspired scientists to generate self-cleaning antireflection coatings (ARCs). ${ }^{10,11}$

Two approaches have been widely exploited for fabricating broadband ARCs. Nanoporous films prepared by layerby-layer deposition of polyelectrolyte or polyelectrolytecolloid multilayers, ${ }^{12,13}$ sol-gel process, ${ }^{14}$ oblique-angle deposition, ${ }^{15}$ colloidal assembly, ${ }^{11,16}$ and nanophase separation of polymer blends, ${ }^{17,18}$ among others, have been developed to fulfill the low refractive index requirement $(\sim 1.2-1.3)$ for efficient ARCs on common glass and plastic substrates. In the second approach, biomimetic moth-eye ARCs with graded refractive index layers can greatly suppress reflection over a broad range of wavelengths and angles of incidence. ${ }^{5,7,8}$ However, current top-down lithographic technologies (e.g., photolithography and interference lithography) for fabricating subwavelength-structured motheye ARCs are expensive and are limited by either low resolution or small sample size.

We have recently developed a simple yet scalable templating technique for fabricating wafer-scale polymer motheye ARCs. ${ }^{19}$ In this approach, a poly(dimethylsiloxane)

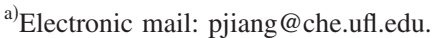

(PDMS) mold is used to transfer the long-range periodic surface protrusions of ncp colloidal crystals prepared by a spincoating process to various polymers. Unfortunately, the intrinsic hydrophilic nature of common nonfluorinated polymers, such as poly(ethoxylated trimethylolpropane triacrylate) (PETPTA) used in our previous study ${ }^{19}$ with an apparent water contact angle of $49.5^{\circ}$, impedes the ultimate realization of self-cleaning ARCs. Here, we extend the templating technique to perfluoroacrylate polymers that possess both low surface energy and low refractive index (RI). The effect of the size and ordering of the replicated nipples on the antireflection performance will also be investigated by experiment and theory.

Although a few perfluorinated monomers (e.g., $1 H, 1 H$, $2 \mathrm{H}, 2 \mathrm{H}$-perfluorodecyl acrylate) are commercially available, many of them form brittle, waxy solids after polymerization, not appropriate for coating applications. ${ }^{20,21}$ We therefore synthesize UV-curable perfluoroether acrylates, such as UV- $T$ with molecular structure shown in Fig. 1, by following the established synthetic procedures. ${ }^{21}$ These multifunctional fluoroacrylate monomers are liquids, allowing solvent-free processing, and the resulting polymers are highly flexible due to their extremely low glass transition temperatures, $T_{g}$

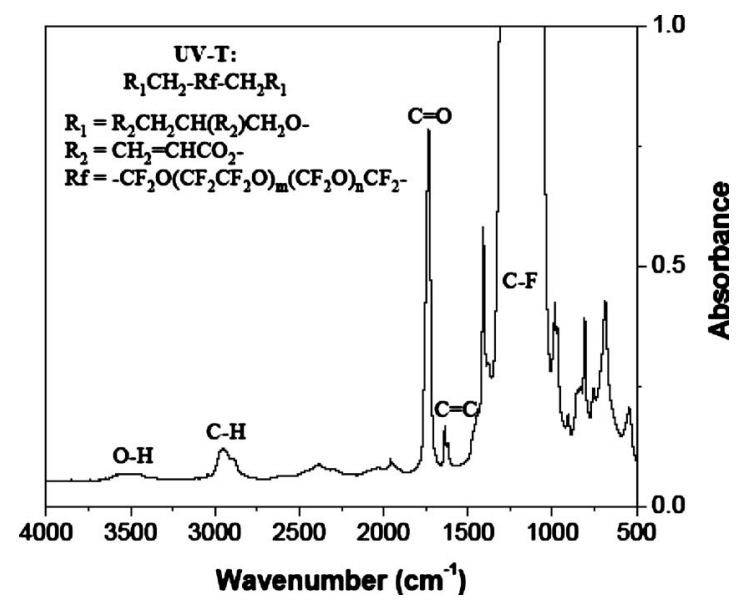

FIG. 1. FTIR spectrum of perfluoropolyether tetraacrylate, UV-T. The molecular structure of UV-T is also shown. 

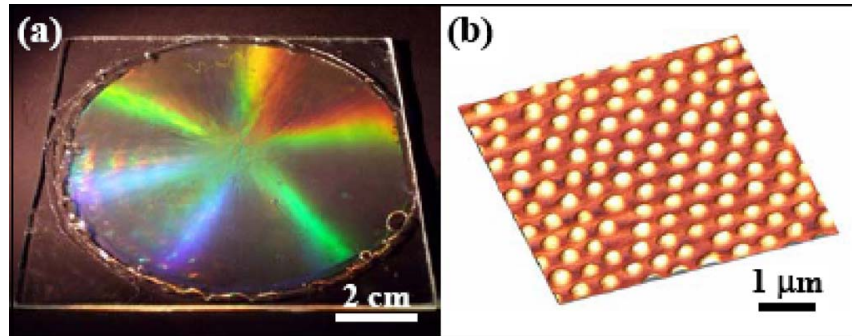

(c)

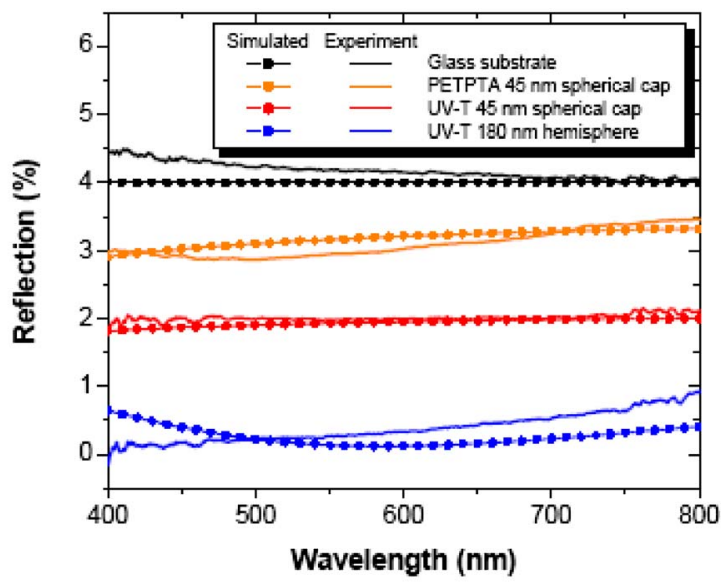

FIG. 2. (Color online) (a) Photograph of a replicated UV-T nipple array on a 4 in. size glass substrate illuminated with white light. (b) AFM image of a nipple array with $\sim 100 \mathrm{~nm}$ height. (c) Experimental (solid) and simulated (dotted) specular optical reflectivity at normal incidence. Black: flat glass substrate. Orange: nonfluorinated polymer (PETPTA) nipple array with $45 \mathrm{~nm}$ height. Red: UV-T nipple array with $45 \mathrm{~nm}$ height. Blue: hemispherical UV-T nipple array with $180 \mathrm{~nm}$ height. All samples are templated from $360 \mathrm{~nm}$ diameter silica spheres.

$\left(\sim-90{ }^{\circ} \mathrm{C}\right) .^{21}$ Fourier transform infrared (FTIR) spectrum for UV-T is shown in Fig. 1 and the absorption bands match with the reported vibrations. ${ }^{21}$ The measured refractive index of UV-T is 1.336 .

We then replicate fluoropolymer nipple arrays on glass substrates by following similar templating procedures, as reported in our previous publication. ${ }^{19}$ To enhance the adhesion of fluorinated UV-T on glass, 3-acryloxypropyl trichlorosilane, which could form covalent linkage between glass and fluoroacrylate polymers, is used to prime the substrates before the photopolymerization of UV-T monomers. ${ }^{22}$ Figure 2(a) shows a photograph of a 4 in. size UV-T nipple array sample templated from $360 \mathrm{~nm}$ silica spheres under white light illumination. The sample exhibits angle-dependent colors caused by Bragg diffraction of visible light by the periodic surface gratings. ${ }^{22}$ The hexagonal ordering and the nonclose packing of templated nipples are clearly evident from the atomic force microscope (AFM) image shown in Fig. 2(b). The protrusion depth of the replicated nipples can be easily controlled by a simple oxygen plasma etching process. ${ }^{19}$

The specular optical reflectivity of the replicated UV-T nipple arrays are evaluated using visible-near-IR reflectivity measurement and then compared with a thin-film multilayer model. ${ }^{19}$ Figure 2(c) compares the measured (solid lines) and simulated (dotted lines) specular reflectivity for a flat glass substrate, a nonfluorinated PETPTA nipple array with $45 \mathrm{~nm}$ spherical caps, a UV-T nipple array with $45 \mathrm{~nm}$ spherical caps, and a UV-T nipple array with $180 \mathrm{~nm}$ hemispherical caps. The flat glass substrate shows $\sim 4 \%$ single-surface nor-

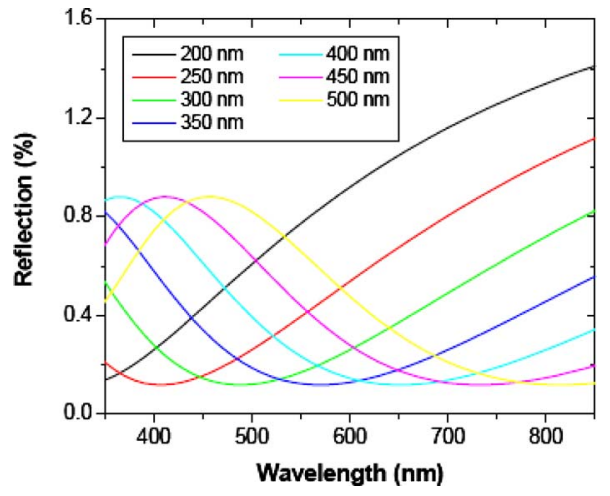

FIG. 3. (Color online) Simulated specular reflectivity at normal incidence for UV-T nipple arrays templated from silica particles with diameters ranging from 200 to $500 \mathrm{~nm}$.

mal reflection (black solid line). When the glass substrate is covered by a nonfluorinated PETPTA $(\mathrm{RI}=1.46)$ nipple array with $45 \mathrm{~nm}$ spherical caps, the reflectivity is reduced to $\sim 3 \%$ (orange solid line). Further improvement is observed when fluorinated UV-T $(\mathrm{RI}=1.336)$ replaces PETPTA. For a UV- $T$ nipple array templated from the same PDMS mold, the normal reflection is only $\sim 2 \%$ (red solid line). Similar improvement is observed for nipples with other depths. The reflectivity progressively decreases with increased nipple height and the reflection from a UV-T hemispherical nipple array (blue solid line) is below $0.5 \%$ for most of the visible spectrum $(400-700 \mathrm{~nm})$. Figure 2(c) also shows that the simulated spectra agree well with the experimental spectra.

We further investigate the size effect of the templated UV- $T$ nipples on the resulting antireflection performance by using the thin-film multilayer model. ${ }^{19}$ Figure 3 shows the simulated specular reflection from seven hemispherical nipple arrays templated from silica particles with diameters ranging from 200 to $500 \mathrm{~nm}$. For small hemispherical nipples (templated from 200 and $250 \mathrm{~nm}$ particles), the reflection is relatively high at long wavelengths; while for large hemispherical nipples (templated from 450 and $500 \mathrm{~nm}$ particles), high reflection occurs at short wavelengths. The same trend has also been confirmed for nonfluorinated polymers with higher refractive indices.

As the natural nipple arrays on moths' corneas exhibit long-range hexagonal ordering, ${ }^{6}$ we continue to investigate the dependence of the crystalline ordering of the templated nipple arrays on the final antireflection properties. For a fair comparison between ordered and disordered nipple arrays, the size, height, and number density of the templated nipples need to be the same. It is straightforward to keep the same size and height of the replicated nipples. ${ }^{19}$ However, maintaining the same number density for both ordered and disordered nipple arrays is not a trivial task. We resolve this difficulty by controlling the oxygen plasma etching process during the removal of the polymer matrix of spin-coated nanocomposites. It is known that there is a $\sim 100 \mathrm{~nm}$ polymer wetting layer between the shear-aligned silica colloidal monolayer and the substrate. ${ }^{23}$ When the oxygen plasma etching time is short [ $<5 \mathrm{~min}$ at $100 \mathrm{mTorr}$ oxygen pressure, 40 SCCM (SCCM denotes cubic centimeter per minute at STP) flow rate, and $100 \mathrm{~W}$ ], the wetting polymer can support the above silica particles and the resulting colloidal crystal is ordered [Fig. 4(a)]; while for longer etching duration, the polymer supporting layer is shrunk significantly and the 


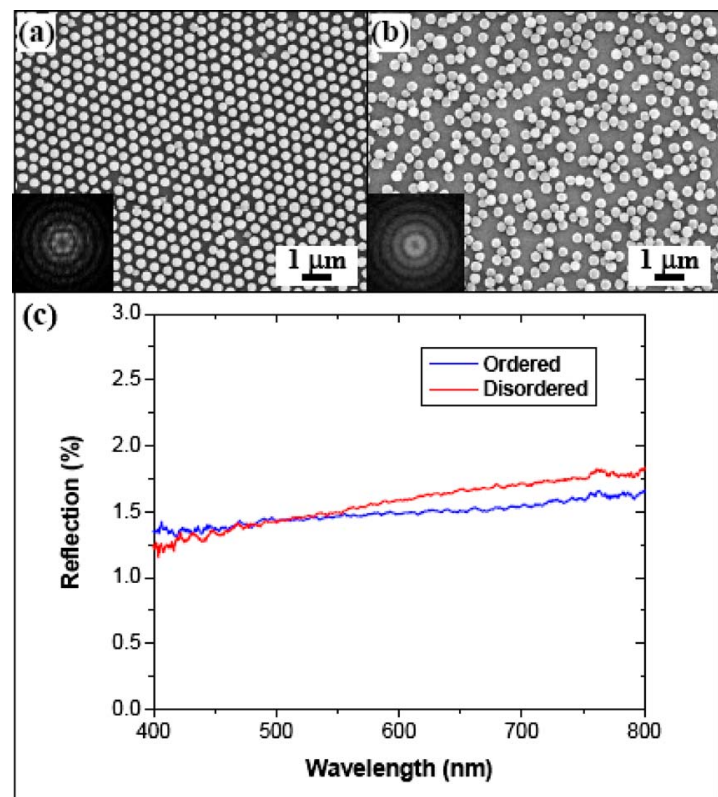

FIG. 4. (Color online) (a) Scanning electron microscope (SEM) image of an ordered colloidal monolayer template. Inset showing Fourier transform of the image. (b) SEM image of a disordered template with the same particle number density as (a). Inset showing Fourier transform of the image. (c) Comparison of the measured normal-incidence specular reflectivity between disordered and ordered UV-T nipples with the same size, height, and number density.

silica particles fall down to form disordered colloidal template [Fig. 4(b)]. By this way, ordered and disordered polymer nipple arrays with exactly the same number density can be fabricated.

Figure 4(c) compares the normal-incidence specular reflectivity spectra between an ordered and a disordered UV-T nipple arrays with the same size $(310 \mathrm{~nm})$, height $(\sim 60 \mathrm{~nm})$, and number density. It is evident that the measured spectra are close to each other. This indicates that the crystalline ordering does not play a crucial role in determining the antireflective properties of the moth-eye ARCs. This result is not too surprising considering the effective medium theory and the thin-film multilayer model. ${ }^{24}$ In the latter model, the nipple arrays are divided into multiple layers and the effective refractive index for each layer is then calculated based on the effective medium theory. As the ordered and disordered arrays have the same number density of nipples and the only difference is their location, the effective refractive index for each layer is thus the same. This leads to the same calculated reflectance from the stack of thin layers.

The utilization of perfluoroether acrylate polymers (e.g., UV-T) as the materials for templating moth-eye ARCs has a number of advantages over nonfluorinated polymers. First of all, the low refractive indices of fluoropolymers coupled with the templated moth-eye microstructures improve the antireflective performance. Second, the elastomeric properties of low- $T_{g}$ fluoropolymers enable the creation of highperformance ARCs on curved surfaces. Most importantly, fluoropolymers are intrinsically hydrophobic, facilitating the ultimate realization of self-cleaning ARCs. A goniometer (NRL C.A. Goniometer, Ramé-Hart, Inc.) is used to measure the apparent water contact angle (CA) on replicated fluoropolymer nipple arrays. The water CA is increased from $\sim 106^{\circ}$ for a flat UV-T film to $\sim 128^{\circ}$ for a hemispherical UV- $T$ nipple array templated from $360 \mathrm{~nm}$ silica spheres. To achieve superhydrophobic ARCs with water $\mathrm{CA}>150^{\circ}$, the aspect ratios of the replicated nipples need to be significantly increased to enhance the fraction of trapped air between nipples. 9,25

In conclusion, we have developed a scalable bottom-up approach for producing fluoropolymer multifunctional optical coatings that simultaneously mimic the functionalities of antireflective moth eyes and superhydrophobic cicada wings. The depth and size of the templated nipples play an important role in determining the antireflective properties of motheye ARCs, while the crystalline ordering has only minimal effect on the reflectivity.

This work was supported in part by the NSF under Grant No. CBET-0651780, the start-up funds from the University of Florida, and the UF Research Opportunity Incentive Seed Fund.

${ }^{1}$ J. Aizenberg, D. A. Muller, J. L. Grazul, and D. R. Hamann, Science 299, 1205 (2003).

${ }^{2}$ K. H. Jeong, J. Kim, and L. P. Lee, Science 312, 557 (2006).

${ }^{3}$ R. A. Potyrailo, H. Ghiradella, A. Vertiatchikh, K. Dovidenko, J. R. Cournoyer, and E. Olson, Nat. Photonics 1, 123 (2007).

${ }^{4}$ P. Vukusic and J. R. Sambles, Nature (London) 424, 852 (2003).

${ }^{5}$ P. B. Clapham and M. C. Hutley, Nature (London) 244, 281 (1973).

${ }^{6}$ D. G. Stavenga, S. Foletti, G. Palasantzas, and K. Arikawa, Proc. R. Soc. London, Ser. B 273, 661 (2006).

${ }^{7}$ A. Gombert, W. Glaubitt, K. Rose, J. Dreibholz, B. Blasi, A. Heinzel, D. Sporn, W. Doll, and V. Wittwer, Thin Solid Films 351, 73 (1999).

${ }^{8}$ U. Schulz, Appl. Opt. 45, 1608 (2006).

${ }^{9}$ T. L. Sun, L. Feng, X. F. Gao, and L. Jiang, Acc. Chem. Res. 38, 644 (2005).

${ }^{10}$ X. F. Gao, X. Yan, X. Yao, L. Xu, K. Zhang, J. H. Zhang, B. Yang, and L. Jiang, Adv. Mater. (Weinheim, Ger.) 19, 2213 (2007).

${ }^{11}$ B. G. Prevo, E. W. Hon, and O. D. Velev, J. Mater. Chem. 17, 791 (2007).

${ }^{12}$ F. C. Cebeci, Z. Z. Wu, L. Zhai, R. E. Cohen, and M. F. Rubner, Langmuir 22, 2856 (2006).

${ }^{13}$ J. Hiller, J. D. Mendelsohn, and M. F. Rubner, Nat. Mater. 1, 59 (2002).

${ }^{14}$ D. G. Chen, Sol. Energy Mater. Sol. Cells 68, 313 (2001).

${ }^{15}$ J. Q. Xi, M. F. Schubert, J. K. Kim, E. F. Schubert, M. F. Chen, S. Y. Lin, W. Liu, and J. A. Smart, Nat. Photonics 1, 176 (2007).

${ }^{16}$ Y. Zhao, J. S. Wang, and G. Z. Mao, Opt. Lett. 30, 1885 (2005).

${ }^{17}$ S. Walheim, E. Schaffer, J. Mlynek, and U. Steiner, Science 283, 520 (1999).

${ }^{18}$ M. Ibn-Elhaj and M. Schadt, Nature (London) 410, 796 (2001).

${ }^{19}$ N. C. Linn, C. H. Sun, P. Jiang, and B. Jiang, Appl. Phys. Lett. 91, 101108 (2007).

${ }^{20}$ J. Samuel, R. Steger, G. Birkle, R. Zengerle, P. Koltay, and J. Ruhe, Anal. Chem. 77, 6469 (2005).

${ }^{21}$ L. W. Shacklette, R. Blomquist, J. M. Deng, P. M. Ferm, M. Maxfield, J. Mato, and H. Zou, Adv. Funct. Mater. 13, 453 (2003).

${ }^{22}$ P. Jiang and M. J. McFarland, J. Am. Chem. Soc. 126, 13778 (2004).

${ }^{23}$ P. Jiang, Chem. Commun. (Cambridge) 2005, 1699.

${ }^{24}$ H. A. Macleod, Thin-Film Optical Filters, 3rd ed. (Institute of Physics, Bristol, 2001).

${ }^{25}$ C. W. Guo, L. Feng, J. Zhai, G. J. Wang, Y. L. Song, L. Jiang, and D. B. Zhu, ChemPhysChem 5, 750 (2004). 
Applied Physics Letters is copyrighted by the American Institute of Physics (AIP). Redistribution of journal material is subject to the AIP online journal license and/or AIP copyright. For more information, see http://ojps.aip.org/aplo/aplcr.jsp 